\title{
Propuesta de Responsabilidad Social Universitaria. Estudio de Caso Chileno, Lecciones por Aprender
}

\author{
Proposal of University Social Responsibility \\ Chilean Case, Lessons to be Learned
}

\begin{abstract}
RESUMEN
En el contexto de la problemática social existente: pobreza (hambre, enfermedades, inseguridad, racismo, migración), contaminación del planeta: aire, el agua, el suelo, situaciones de violencia en la comunidad y otras formas de degradación y daño social, la Universidad tiene que asumir su responsabilidad tal como lo establece la Constitución Política del Perú, la ley Universitaria 30220, las disposiciones internacionales establecidas en la Red de Pacto Mundial que comprometen la adhesión de instituciones al cumplimiento de sus principios y lineamientos de respeto a los Derechos Humanos, los Derechos Laborales, el Medioambiente, la ISO 26000, la Norma SA8000, Social Accountability AA1000, la Global University Network for Innovation-GUNI (UNESCO-UNU-ACUP).

La presente propuesta tiene como objetivos el de determinar las estrategias para una acertada Responsabilidad Social Universitaria (R.S.U.), establecer la importancia de los medios de comunicación y la Tecnologías de la Información y Comunicación en el logro de la R.S.U. y reconocer el valor de las políticas institucionales en la implantación de una Cultura socialmente responsable en el contexto universitario que tendrán un gran impacto en la productividad, la satisfacción de los recursos humanos, el cuidado y preservación del capital natural y el fortalecimiento del compromiso institucional.
\end{abstract}

Palabras claves: Responsabilidad Social Universitaria; estrategias; políticas institucionales.

\begin{abstract}
In the context of the existing social problems: poverty (hunger, diseases, insecurity, racism, and migration), pollution of the planet: air, water, soil, situations of violence in the community and other forms of degradation and social damage. The University must assume its responsibility as established by the Political Constitution of Peru, University Law 30220. International regulations established in the Global Compact Network that commit the adherence of institutions to compliance with its principles and guidelines of respect for Human Rights , Labor Rights, the Environment, ISO 26000, Standard SA8000, Social Accountability AA1000, the Global
\end{abstract}

\footnotetext{
${ }^{1}$ Licenciada en Educación, egresada de la Maestría en Administración, gestión de Recursos Humanos y Doctoranda en Medio Ambiente y Desarrollo Sostenible. Concesión de Becas de Movilización para Investigación por la UIM, España, Universidad Nacional de Quilmes (Argentina) y Universidad Santiago de Cali (Colombia), Certificado de participación en la Reducción de Riesgo de Desastres y Desarrollo Local Sostenible en OIT, Turin (Italia). Producción Científica en ORCID, DINA-CONCYTEC. Asistencia a eventos empresariales en Casa Piedra, Santiago de Chile y participación en Centro de Referencia Copernicus, Centro de Modelamiento Matemático de la Universidad de Chile.
}

(C) Los autores. Este artículo es publicado por Gestión en el Tercer Milenio de la Facultad de Ciencias Administrativas de la Universidad Nacional Mayor de San Marcos. Este es un artículo de acceso abierto, distribuido bajo los términos de la licencia Creative Commons Atribucion - No Comercia_Compartir Igual 4.0 Internacional. (http://creativecommons.org/licenses/by-nc-sa/4.0/) que permite el uso no comercial, distribución y reproducción en cualquier medio, siempre que la obra original sea debidamente citada. 
University Network for Innovation-GUNI (UNESCO-UNU-ACUP).

The objective of this proposal is to determine the strategies for a successful University Social Responsibility (R.S.U.). Establish the importance of the media, Information, and Communication Technologies in achieving the R.S.U. And to recognize the value of institutional policies in the implementation of a socially responsible culture in the university context that will have a great impact on productivity, the satisfaction of human resources, the care and preservation of natural capital and the strengthening of institutional commitment.

Keywords: University Social Responsibility, strategies, institutional policies.

\section{INTRODUCCIÓN}

Ante la problemática social existente: pobreza (hambre, enfermedades, inseguridad, violencia, racismo, migración), contaminación del planeta ante la contaminación del aire, el agua, el suelo) y situaciones de violencia en la comunidad es de vital importancia que se garantice la paz y prosperidad sociales, la disminución de la pobreza y la protección y cuidado ambiental: aire, agua, suelo y otras formas de degradación y protección social. En este contexto la Universidad, como organización de la sociedad, tiene que asumir su responsabilidad tal como lo establece la Constitución Política del Perú, la ley Universitaria 30220, las disposiciones internacionales establecidas en la Red de Pacto Mundial que compromete la adhesión de instituciones al cumplimiento de sus principios y lineamientos de respeto a los Derechos Humanos, los Derechos Laborales, el Medioambiente y el desarrollo.

Asimismo, lo indica la ISO 26000 que ofrece orientaciones fundamentales de responsabilidad social en la universidad en el ámbito gerencial, transformacional y normativo dirigido a estudiantes, personal docente, personal de investigación, personal de la administración y servicios en jornadas de sensibilización, talleres, reuniones

Además, la Norma SA8000, Social Accountability 8000 establece pautas de ética para las empresas y sus trabajadores y las normas Accountability AA1000 que garantiza la calidad de las rendiciones de cuentas, evaluaciones y divulgación en las instituciones frente a los diversos problemas que constituye un reto para la sociedad permitiendo el acercamiento de la universidad y resolverlos.
A través de un análisis renovador de los principales temas de educación superior, La Global Universitiy Network for Innovation-GUNI (UNESCO-UNU-ACUP) conformada por miembros de los cinco continentes, la Universidad de las Naciones Unidas y la Asociación de Universidades Públicas Catalanas (ACUP), contribuye al fortalecimiento del rol de la educación superior en la sociedad.

Por otro lado, la Declaración de Talloires (Red Talloires) dedicada al fortalecimiento de los roles cívicos en la educación superior compromete a las instituciones firmantes, las universidades, a emplear procesos educativos y de investigación para responder, servir y fortalecer a la ciudadanía en los ámbitos local y global para contribuir a una cultura de la reflexión y la acción. Otras tareas académicas de la universidad como los proyectos (Dridiksson, 2007) promueven la cooperación e intercambio de experiencias docentes en investigación y extensión de la cultura y la formación con valores.

Finalmente, la UNESCO desde el año 1998 viene planteando que las universidades a nivel mundial deben asumir una mayor responsabilidad social dentro de la denominada sociedad del conocimiento, con su rol como instituciones especializadas en la generación transmisión del conocimiento científico.

\section{DEFINICIONES DE RESPONSABILIDAD SOCIAL UNIVERSITARIA}

Importantes aportes de investigadores han contribuido a una mayor comprensión de la Responsabilidad Social Universitaria, los que a continuación se mencionan:

La definición de Vallaeys (2007) ha ganado aceptación y credibilidad: 'una política de gestión de la calidad ética de la Universidad que 
busca alinear sus cuatro procesos (gestión, docencia, investigación, extensión) con la misión universitaria, sus valores y compromiso social, mediante el logro de la congruencia institucional, la transparencia y la participación dialógica de toda la comunidad universitaria (autoridades, estudiantes, docentes, administrativos) con los múltiples actores sociales interesados en el buen desempeño universitario para lograr la transformación efectiva de la sociedad hacia la solución de sus problemas de exclusión, inequidad y sostenibilidad. Vallaeys indica seguir un proceso para la implementación de la RSU consistente en el Compromiso según la misión y valores de la institución, el Autodiagnóstico, el Cumplimiento y la Rendición de Cuentas.

Wigmore Alvarez (2016) Gestión de la Responsabilidad Social Universitaria, explica que los cambios sociales, ambientales y tecnológicos de los últimos tiempos han logrado que las organizaciones consideren su responsabilidad por que han empezado a asumir su responsabilidad social con ideas creativas y colaborativas ante los diferentes cambios sociales, ambientales y tecnológicos. De allí que las universidades no pueden permanecer indiferentes por lo que están aplicando una serie de modelos de implementación de lineamientos establecidos en la Agenda 2030 con sus 17 objetivos que complementan los anteriores Objetivos del Milenio del 2005 al 2014, Década del Desarrollo Sostenible. A través de la responsabilidad social, los estudiantes establecen contacto real con la problemática de la sociedad lo que alimenta su proceso de formación profesional y se desarrolla el futuro profesional y dará respuesta a las demandas y exigencias del entorno.

Benavides Velasco, Carlos (2008) indica que el proceso de construcción de una agenda en ciencia, tecnología e innovación compartida por la universidad latinoamericana y caribeña debe apuntar a generar el conocimiento de nuestro desarrollo y bienestar de nuestros pueblos y demanda y propiciar una creciente comprensión de la ciencia como un asunto público que concierne a la sociedad den su conjunto.

Castañeda et al. (2007) señala que la gestión de una política de investigación dirigida a una gestión social del conocimiento participativo: miembros de la comunidad, estudiantes y docentes, permite la adquisición de conocimientos socialmente útiles para todos. La RTSU se encamina a fomentar el desarrollo sostenible y la resolución de problemas concretos que afectan la sociedad y promover el aprendizaje comunitario realizando alianzas estratégicas con otras instituciones de la sociedad para la mejora de las prácticas profesionales y sociales, y permitan la interacción de instituciones públicas y privadas del país.

Larra y López (2010) firman que la investigación sobre la RSU ha estado relacionada con temas del quehacer universitario: la evaluación de las percepciones, análisis de la oferta formativa sobre responsabilidad social corporativa en las universidades.

Ojeda (2013) analiza la percepción de los profesores universitarios sobre el concepto de RSU en contraste con lo que propone ISO 26000. Los docentes señalaron las dimensiones de medio ambiente, derechos humanos y el involucramiento con la comunidad.

Hill, 2004, Mehta, 2011: han estudiado el aporte de la RSU para promover una mejor relación de la universidad con la comunidad local.

Aristimuño y Rodríguez (2014) analizan las dimensiones subyacentes de la RSU en una universidad venezolana, destacando a los directivos y docentes como los más importantes tomando en cuenta el comportamiento organizacional, la selección de los stakeholders y la planificación hacia el comportamiento social.

Llopis, 2010: debería orientarse a tomar papel activo en la difusión de la CULTURA para que se genere pensamiento crítico entre la comunidad universitaria y el resto de la universidad y que abra sus puertas a todas las personas (Cuadro 1).

\section{La Responsabilidad Social universitaria en el entorno Europeo y Latinoamericano}

El acercamiento Universidad - Sociedad requiere de estrategias diversas acorde a sus diferentes funciones en la formación, investigación y proyección social, que se mencionan en cuadro adjunto de diferentes países europeos y latinoamericanos cuyo practica puede útil en la implantación de dichas estrategias en nuestras instituciones (Cuadro 2). 
Cuadro 1.

Normativas que establecen la Responsabilidad Social Universitaria

\begin{tabular}{|c|c|c|}
\hline INSTITUCION/ AUTOR & EVENTO & ENLACE \\
\hline $\begin{array}{l}\text { Universidad Internacional de } \\
\text { Catalunya } \\
\text { R.S.U. en la formación curricu- } \\
\text { lary participación social }\end{array}$ & $\begin{array}{l}\text { Entre el siglo XV y XIV tras la Revolución Industrial, y la crisis } \\
\text { de } 1929 \text {, surgen las leyes que explicitan el papel de las empre- } \\
\text { sas para mejorar el bienestar social. } \\
\text {-En } 1945 \text { se crea la ONU para mantener la paz y promover el }\end{array}$ & $\begin{array}{l}\text { https://www.tdx.cat/bitstream/ } \\
\text { handle/10803/283417/Tesi_ } \\
\text { Azul_Alf\%C3\%A9rez_Villareal. } \\
\text { pdf?sequence=1\&isAllowed=y }\end{array}$ \\
\hline
\end{tabular}

Azul Alférez Villarreal 2014

Red Chilena 'Universidad construye País

Protocolo de Kioto

El Global Reporting Initiative (GRI)

La publicación ISO SA8000 de R.S.

El Libro Verde de la Comisión Europa (1999

El Global Compact o Pacto Mundial (2000) iniciativa internacional de la Organización de las Naciones Unidas

La Cumbre del Milenio (2000) que desarrolla los Objetivos del Milenio

El Libro Blanco de la Comisión Europea

Resolución del Parlamento Europeo sobre la RS de las empresas (2007) progreso social y contribuye a la Declaración de los Derechos Humanos en 1948

-en la década de los 60 y 70 aumenta la inquietud por los temas sociales y en 1961 se crea la World Wildlife Fund (WWF) para combatir la contaminación

-1987 surge el Informe Brundtland de la Comisión Mundial sobre medioambiente de la ONU y aparece el término Desarrollo Sostenible.

-En la década de los ochenta comienza la globalización mercantil y la Responsabilidad Social complementa el Desarrollo Sostenible y se consolida en 1989

A inicios de los años 2000 se construyó explícitamente el concepto de responsabilidad social universitaria (RSU) en América Latina, alrededor de los esfuerzos teóricos y prácticos de la Red chilena "Universidad Construye País" y de la red Latinoamericana de Universidades animada por la "Iniciativa Interamericana de Ética, Capital Social y Desarrollo", promovida por el gobierno noruego en el seno del Banco Interamericano de Desarrollo (BID) y que dejó de funcionar en 2009.

Se explicitan responsabilidades medioambientales que busca reducir las emisiones de gases de efecto invernadero en el planeta y promueve el crecimiento sustentable de los países en desarrollo

Establece unos indicadores en temas de RSU. Empresas y organizaciones se juntan para mejorar sus procesos de negocios y ayudan a un futuro sostenible

Establece indicadores para valorar la RS a través de entidades externas que emiten un certificado de evaluación

Marca las líneas directrices de la OCDE (Organización para la Cooperación y el Desarrollo Económico) y fomenta un marco europeo para la responsabilidad social de las empresas

Insta a las empresas a comprometerse con los principios de desarrollo sostenible y marca una alianza global para e 2030 para las empresas sostenibles: Objetivos del Desarrollo Sostenible: el 25 setiembre 2015, los 17 objetivos del desarrollo sostenible de la Agenda 2030 fueron adoptados por 193 países en la histórica cumbre de Paris.

Algunos objetivos son: combatir la pobreza, el hambre, las enfermedades, el analfabetismo, el daño ambiental, el analfabetismo, daño al medioambiente, discriminación de la mujer. Se difunden valores de libertad, igualdad, solidaridad, tolerancia, respeto a la naturaleza.

Expone temas relacionados al medioambiente y las responsabilidades sociales, sistema de responsabilidad ambiental para prevenir y reparar daños. Reflexiones y escenarios para la Europa de los Veintisiete en 2025

Considera que las empresas podrían apadrinar actividades culturales y educativas, incentiva la política de RS para realizar inversiones responsables para combatir la pobreza y fomentar condiciones dignas para el comercio justo y buen gobierno. Se promueve la dimensión internacional de la RS para fomentar líneas de políticas mundiales www.sciencedirect.com/science/ article/pii/S2007287214719456

https://www.gob.mx/semarnat/ articulos/protocolo-de-kioto-sobre-cambio-climatico?idiom $=\mathrm{es}$

https://www.globalreporting. org/Pages/default.aspx

http://www.fao.org/docrep/007/ ad818s/ad818s06.htm

observatoriorsc.org/libro-verde-fomentar-un-marco-europeo-para-la-responsabilidad-social-de-las-empresas/

https://www.pactomundial.org/

http://www.un.org/es/development/devagenda/millennium. shtml

https://ec.europa.eu/commission/sites/beta-political/files/ libro_blanco_sobre_el_futuro_de europa_es.pdf

https://www.economiasolidaria.org/biblioteca/ resolucion-del-parlamento-europeo-favor-del-emprendimiento-social-y-las-empresas 
Cuadro 2.

\begin{tabular}{|c|c|c|c|}
\hline ENTIDAD PARTICIPANTE & FUENTE & ACCIONES & BENEFICIARIOS \\
\hline Universidad Mayor & $\begin{array}{l}\text { https://www.umayor.cl/ } \\
\text { dae-umayor/trabajos-voluntar- } \\
\text { ios.php }\end{array}$ & $\begin{array}{l}\text { Trabajo voluntariado para } \\
\text { contribuir a la formación y ad- } \\
\text { quisición de la responsabilidad } \\
\text { social de los estudiantes }\end{array}$ & $\begin{array}{l}14 \text { mil personas vulnerables } \\
\text { de la región Coquimbo }\end{array}$ \\
\hline $\begin{array}{l}\text { Universidad Autónoma de } \\
\text { Chile }\end{array}$ & $\begin{array}{l}\text { https://www.uautonoma.cl/ } \\
\text { vinculacion-con-el-medio/ } \\
\text { programas-y-mecanismos-de- } \\
\text { la-vme/responsabilidad-so- } \\
\text { cial-y-voluntariado/ }\end{array}$ & $\begin{array}{l}\text { Redes de colaboración y Volun- } \\
\text { tariado } \\
\text { http://voluntariado.uautono- } \\
\text { ma.cl/ }\end{array}$ & $\begin{array}{l}\text { Personas con discapacidad } \\
\text { Niños oxigeno dependientes }\end{array}$ \\
\hline Universidad Andrés Bello & Diario El Mercurio (23.08.18) & $\begin{array}{l}\text { Estudio del fondo marino, } \\
\text { lechos del subsuelo marino }\end{array}$ & Entorno social, ecosistema \\
\hline $\begin{array}{l}\text { Universidad Católica de } \\
\text { Valparaíso }\end{array}$ & $\begin{array}{l}\text { http://www.cona.cl/publi- } \\
\text { caciones/geologia_marina/ } \\
\text { editorial.pdf }\end{array}$ & $\begin{array}{l}\text { Trabajos de investigación, pub- } \\
\text { licaciones, grupos de trabajo }\end{array}$ & Fondos marinos de Chile \\
\hline Universidad de Chile & $\begin{array}{l}\text { https://www.biobiochile.cl/ } \\
\text { noticias/ciencia-y-tecnologia/ } \\
\text { ciencia/2018/08/13/cua- } \\
\text { tro-proyectos-de-geologia-inte- } \\
\text { graran-el-primer-crucero-geo- } \\
\text { logico-de-chile.shtml }\end{array}$ & $\begin{array}{l}\text { Proyectos de Geología marina, } \\
\text { talud continental, placas, } \\
\text { cánones submarinos }\end{array}$ & $\begin{array}{l}\text { Entre Puerto Montt y la } \\
\text { Península de Taitao }\end{array}$ \\
\hline Universidad San Sebastián & Publimetro 17.08 .18 & Estudio de huellas dactilares & Adultos mayores de Santiago \\
\hline Universidad Santo Tomas & El Mercurio 29.08.18 & $\begin{array}{l}\text { Hay centros de Investigación en } \\
\text { colaboración con El Mercurio }\end{array}$ & $\begin{array}{l}\text { Antofagasta y Tierra de Fuego } \\
\text { al sur de Chile }\end{array}$ \\
\hline Universidad Mayor & $\begin{array}{l}\text { Cambio Empresas } \\
\text { Enlace: }\end{array}$ & $\begin{array}{l}\text { Alimentación saludable en } \\
\text { convenio con Municipalidad }\end{array}$ & $\begin{array}{l}\text { Escuelas kínder y primarias } \\
\text { desde Arica a Punta Arenas }\end{array}$ \\
\hline Universidad de Chile & $\begin{array}{l}\text { https://www.youtube.com/ } \\
\text { watch?v=9-6DVZQbagc }\end{array}$ & $\begin{array}{l}\text { U. de Chile inauguró Campo } \\
\text { Deportivo de } 9 \text { mil metros } \\
\text { cuadrados en el Campus Juan } \\
\text { Gómez Millas }\end{array}$ & Universidad Prensa Chile \\
\hline $\begin{array}{l}\text { Universidad del Desarrollo } \\
\text { UDD. Triple Hélice para } \\
\text { creación de valor en empresas }\end{array}$ & Cambio Empresas & $\begin{array}{l}\text { Triple Hélice: Telefónica y em- } \\
\text { presa Corfo (Estado) }\end{array}$ & $\begin{array}{l}\text { Empresas del Estado } \\
\text { (Minería, Agro, Ciudades } \\
\text { Sostenibles) }\end{array}$ \\
\hline Universidad de Chile & $\begin{array}{l}\text { https://www.youtube.com/ } \\
\text { watch?v=j0ybWQp6rUU } \\
\text { Facultad de Odontología }\end{array}$ & $\begin{array}{l}\text { Teletón y Universidad de Chile } \\
\text { firman convenio para formar a } \\
\text { Odontopediatras }\end{array}$ & $\begin{array}{l}\text { Población niñez universitaria } \\
\text { y público en condición de } \\
\text { discapacidad }\end{array}$ \\
\hline Red Chilena Construye País & $\begin{array}{l}\text { http://www.rsuchile.cl/p/ } \\
\text { universidad.html }\end{array}$ & $\begin{array}{l}\text { Legado del proyecto Universi- } \\
\text { dad Construye País desarrolla- } \\
\text { do en Chile entre } 2001 \text { y } 2008\end{array}$ & A la comunidad en general \\
\hline
\end{tabular}

\section{OBJETIVOS}

1. Determinar las estrategias para una acertada Responsabilidad Social Universitaria.

2. Establecer la importancia de los medios de comunicación y las TICs en el logro de la Responsabilidad Social Universitaria.

3. Reconocer el valor de las políticas institucionales en la implantación de una cultura socialmente responsable en el contexto universitario.

\section{METODOLOGÍA}

Esta investigación es cualitativa, de diseño descriptivo que utilizó estrategias de observación, análisis, consulta, entrevistas aplicados en reciente visita al país vecino de Chile en julio y agosto del presente año, habiendo acudido a muchas fuentes de información documentales, eventos realizados en la comuna de Santiago y alguna de sus instituciones como universidades, centros culturales, centros de investigación, entidades del Estado, empresas, colegios y otros. 
A ello se agrega la investigación en línea, consulta a fuentes bibliográficas en internet sobre cómo se ha ido implantando la Responsabilidad Social Universitaria en Chile acorde a los lineamientos en su constitución, principios y normativas. Se consultó fuente de información escrita de periódicos como La Tercera, El Mercurio de los meses de julio, agosto y setiembre 2018.

Se participó en eventos organizados por instituciones, empresas y universidades figuran el de Acción Empresas, Cambio y Fuera, Protagonistas 2030 llevados a cabo en Casa Piedra, sito en la ciudad de Santiago, con la asistencia y apoyo de empresas como: Biofactorías, Aguas Andinas, Sodimac, Arauco,.Aquachile, Colbun, CEDENNA, Fundación Chile, CNN Chile, CLARO y universidades como Universidad de Chile, Pontificia Universidad Católica de Valparaíso, Universidad del Desarrollo, Universidad Tecnológica de Chile, universidad Mayor, Universidad Andrés Bello, Universidad San Sebastián, Universidad Técnica, Federico Santa María y otras más.

Se elaboró cuadro-resumen de relación de universidades comprometidas con la Sociedad en donde llevan a cabo obras de voluntariado, investigación, formación, estudio, presentación de proyectos, publicaciones, asesorías e importantes acciones de labor social que a continuación se detallan:

\section{RESULTADOS}

1. Aplicación de las 3Ps (Planificación, Participación y Presupuesto) en la consecución de la Responsabilidad Social Universitaria.

2. Labor significativa de los medios de comunicación y las redes sociales de los diarios El Mercurio, La Tercera que cumplen una labor muy importante en la difusión diaria de los eventos de responsabilidad social que llevan a cabo las universidades y otras entidades públicas y privadas.

3. La capacitación de los recursos humanos de las organizaciones chilenas (empresas e instituciones públicas y privadas) que va desde sus directivos, administrativos, personal académico, de servicio, de investigación, docentes, alumnos, exalum- nos permiten establecer una cultura de Responsabilidad Social y se manifiesta en una buena toma de decisiones de sus directivos, conducta responsablemente social de sus futuros profesionales $\mathrm{y}$ egresados, grado de compromiso en la enseñanza e investigación de sus docentes, calidad en el servicio y atención al cliente interno y externo avanzando hacia el fortalecimiento de responsabilidad social dentro y fuera del campus universitario.

\section{CONCLUSIONES}

1. Los elementos que constituyen las 3Ps (Planificación, Participación y Presupuesto) son el resultado de una Información oportuna, precisa y adecuada que brindan los Medios de Información (radio, periódicos, TV, las redes sociales) unido a la aplicación de una sensibilización y concientización de los recursos humanos lo que redunda en su Participación eficaz.

2. Los medios de información y comunicación, TICs masiva como prensa, revistas, noticieros de radio y televisión, páginas web, wasap y otros tienen un rol significativo en la información, educación, capacitación de las organizaciones.

3. La Cultura Organizacional es la clave para el logro de los objetivos trazados y se logra con educación, charlas de capacitación continuos y generan gran impacto en la productividad, satisfacción de los recursos humanos, el cuidado y la preservación del capital natural y el fortalecimiento del compromiso institucional.

\section{REFERENCIAS BIBLIOGRÁFICAS}

Díaz, Julián (2015). Concepciones Sobre La Formación En Responsabilidad Social. Pontificia Universidad Javeriana. Colombia

Carrillo'-Pacheco, Marco (2012). Responsabilidad Social Universitaria. La Opinión De Profesores $Y$ Alumnos. Universidad De Querétaro

Baca-Neglia, Hilda (2017) Propuesta De Medición De La Responsabilidad Social Universitaria. Revista Espacios

Aldea nueva, Ignacio (2011). Responsabilidad Social En La Universidad: Estudio De Casos Y Propuesta De Despliegue. Universidad De Málaga 
Cortes, Gustavo (2012). Responsabilidad Social Universitaria. Una Mirada A La Universidad Nacional De Colombia

Experiencias Iberoamericanas En Responsabilidad Social Universitaria. 2015

Didriksson Axel (2014) La Universidad En La Sociedad Del Conocimiento: Hacia Un Modelo De Producción Y Transferencia De Conocimientos $Y$ Aprendizajes, Unalm, México

Vallaeys, Francois (2007). La Responsabilidad Social Universitaria, Tecnológico De Mexico

Wigmore Alvarez (2016). Gestión De La Responsabilidad Social Universitaria

Benavides-Velasco Carlos (2008). Innovative Competence, Exploration and Exploitation: The Influ- ence of Technological Diversification. University Of Málaga

Larran Manuel (2007) Análisis De Responsabilidad Social Universitaria Desde Diferentes Enfoques Teóricos. Revista Iberoamericana De Educación Superior

Ojeda José (2015) Responsabilidad Social en las Universidades: Antecedentes, Trayectorias y Perspectivas

Vallaeys Francois (2017) Introducción A La Responsabilidad Social Universitaria. Universidad Simón Bolívar.

Aristimuño Y Rodríguez (2014) Responsabilidad Social Universitaria. Su Gestión Desde La Perspectiva De Reactivos Y Docentes. Estudio De Caso. Caracas, Venezuela 
